\title{
Actin Family Proteins in the Human INO80 Chromatin Remodeling Complex Exhibit Functional Roles in the Induction of Heme Oxygenase-1 with Hemin
}

\begin{abstract}
Yuichiro Takahashi ${ }^{1}$, Hirokazu Murakami ${ }^{1}$, Yusuke Akiyama ${ }^{1}$, Yasutake Katoh ${ }^{2}$, Yukako Oma ${ }^{1}$, Hitoshi Nishijima ${ }^{3,4}$, Kei-ichi Shibahara ${ }^{3}$, Kazuhiko Igarashi ${ }^{2,5}$ and Masahiko Harata ${ }^{1 *}$

${ }^{1}$ Laboratory of Molecular Biology, Graduate School of Agricultural Science, Tohoku University, Sendai, Japan, ${ }^{2}$ Department of Biochemistry, Graduate School of Medicine, Tohoku University, Sendai, Japan, ${ }^{3}$ Department of Integrated Genetics, National Institute of Genetics, Mishima, Japan, ${ }^{4}$ Division of Molecular Immunology, Institute of Advanced Medical Sciences, Tokushima University, Tokushima, Japan, ${ }^{5}$ Japan Agency for Medical Research and Development, Tokyo, Japan
\end{abstract}

Nuclear actin family proteins, comprising of actin and actin-related proteins (Arps), are essential functional components of the multiple chromatin remodeling complexes. The INO80 chromatin remodeling complex, which is evolutionarily conserved and has roles in transcription, DNA replication and repair, consists of actin and actin-related proteins Arp4, Arp5, and Arp8. We generated Arp5 knockout (KO) and Arp8 KO cells from the human Nalm-6 pre-B cell line and used these KO cells to examine the roles of Arp5 and Arp8 in the transcriptional regulation mediated by the INO80 complex. In both of Arp5 $\mathrm{KO}$ and Arp8 $\mathrm{KO}$ cells, the oxidative stress-induced expression of HMOX1 gene, encoding for heme oxygenase-1 ( $\mathrm{HO}-1)$, was significantly impaired. Consistent with these observations, chromatin immunoprecipitation (ChIP) assay revealed that oxidative stress caused an increase in the binding of the INO80 complex to the regulatory sites of HMOXI in wild-type cells. The binding of INO80 complex to chromatin was reduced in Arp8 KO cells compared to that in the wild-type cells. On the other hand, the binding of INO80 complex to chromatin in Arp5 $\mathrm{KO}$ cells was similar to that in the wild-type cells even under the oxidative stress condition. However, both remodeling of chromatin at the HMOX1 regulatory sites and binding of a transcriptional activator to these sites were impaired in Arp5 $\mathrm{KO}$ cells, indicating that Arp5 is required for the activation of the INO80 complex. Collectively, these results suggested that these nuclear Arps play indispensable roles in the function of the INO80 chromatin remodeling complex.

Keywords: actin family, chromatin, chromatin remodeling, heme oxygenase-1, stress response

\section{INTRODUCTION}

In the nucleus of eukaryotes, the genomic DNA is packaged into a complex nucleoprotein structure, known as chromatin. Chromatin structure restricts the accessibility of factors involved in the DNAbased cellular process, including transcription, DNA replication, and DNA damage repair. The ATP-dependent chromatin-remodeling complexes regulate these processes through remodeling 
of the chromatin structure. These complexes consist of an enzymatic component and multiple subcomponents, which regulate their complex functions. It is known that some of the chromatin remodeling complexes contain actin family proteins as essential subcomponents (Oma and Harata, 2011).

The actin family consists of conventional actin and actinrelated proteins (Arps). Arps are classified into Arp1 to Arp10 subfamilies, and each subfamily is evolutionarily conserved (Dion et al., 2010; Oma and Harata, 2011). The structure of the core domain of actin and Arp molecules, known as the actin fold, is highly conserved among the members of the actin family (Fenn et al., 2011; Gerhold et al., 2012). On the other hand, each member of this family has distinct molecular surface structure. Whereas some of the Arps are predominantly localized in the cytoplasm, several Arps (namely Arp 4, Arp 5, Arp 6, Arp 7, Arp 8, and Arp 9) are accumulated in the nucleus (Dion et al., 2010; Oma and Harata, 2011). Consistent with these results, all of these nuclear Arps were found to be parts of chromatin remodeling complexes of which actin is also a component.

The INO80 chromatin remodeling complex contains four actin family members: actin, Arp4, Arp5, and Arp8 (Oma and Harata, 2011; Tosi et al., 2013). These actin family proteins of the INO80 complex is evolutionarily conserved from yeast to human (Cai et al., 2007; Wu et al., 2007; Yao et al., 2008). Although actin and Arp4 are also found in some other chromatin remodeling complexes, Arp5 and Arp8 are identified as specific components of the INO80 complex. In addition to containing the Ino80 molecule as a scaffold, the budding yeast INO80 complex also contains four topological modules, namely Arp8-, Arp5-, Nhp10-, and Rvb-modules (Tosi et al., 2013).

Arp8 is included in the Arp8-module together with actin and Arp4, and has histone binding ability (Shen et al., 2003; Gerhold et al., 2012). We have recently reported that the human Arp8 binds to double- and single-stranded DNAs (Osakabe et al., 2014). We have also established gene knockout (KO) human cell line lacking Arp8 (Arp8-KO cells), and showed that Arp8 is required for the double strand DNA break (DSB) repair function of the INO80 complex (Osakabe et al., 2014). However, human Arp8's contribution to transcriptional regulation remains yet to be analyzed using the Arp8KO cells. Although Arp5, which is included in the Arp5-module, a module different from the Arp8module, is supposed to have a distinctive role in the INO80 complex (Kitamura et al., 2015), our knowledge regarding the function of the human Arp5 in the INO80 complex is still very limited, especially its role in transcriptional regulation.

Reactive oxygen species (ROS), which are highly reactive in nature and cause oxidative stress, are known to disturb cellular homeostasis, which in some cases lead to the development of cancer and other diseases (Loboda et al., 2016). ROS are known to induce transcription of a set of genes, and heme oxygenase-1 (HO-1), which is a representative oxidative stress-inducible protein, has potent anti-inflammatory, antioxidative and antiproliferative effects (Vile et al., 1994; Camhi et al., 1995; Maeshima et al., 1996). Recently, Katoh et al. (2011) observed that Ino80 and Arp4 in complexes associate with the promoter of HMOX1 gene, which encodes for HO-1, suggesting a possibility that the INO80 complex is involved in the transcriptional regulation of HMOX1.

In this study, we examined the roles of human Arp5 and Arp8 in the transcriptional regulation mediated by the INO80 complex using respective $\mathrm{KO}$ cells. We observed that these actin family proteins have distinctive roles in the activation of HMOX1. Using ChIP analysis we found that Arp8, but not Arp5, is required for the binding of INO80 complex to chromatin. On the other hand, both remodeling of chromatin at the HMOX1 regulatory sites and binding of a transcription activator to these regulatory sites were impaired in Arp5 KO cells. These observations provided novel information about the distinctive functional contributions of actin family proteins in the transcriptional regulation mediated by the human INO80 complex.

\section{MATERIALS AND METHODS Cell Culture and Induction of Oxidative Stress}

Nalm-6 pre-B cells were cultured at $37^{\circ} \mathrm{C}$ in Roswell Park Memorial Institute medium containing GlutaMAX $^{\mathrm{TM}_{-} \mathrm{I}}$ (Invitrogen) supplemented with $10 \%$ fetal bovine serum, penicillin, and streptomycin as described previously (Ono et al., 2009). To induce oxidative stress, hemin (Sigma) was added to the culture medium to a final concentration of $20 \mu \mathrm{M}$.

\section{Establishment of Arp5-Knockout (KO) Cells}

Arp5 KO cells were generated using protocols similar to that was used for the generation of Arp8 KO cells (Osakabe et al., 2014) with some modifications. Briefly, the left $(3.1 \mathrm{~kb})$ and right $(3.6$ $\mathrm{kb})$ arms of the targeting vectors were, respectively, amplified by PCR using the genomic DNA purified from Nalm-6 pre-B cells as the template. The left arm and the right arm contained exons 2-3 and intron 5, respectively (see Supplementary Figure S1). The disruption of both alleles of the ARP5 gene was confirmed by Southern blot analysis using probes shown in Supplementary Figure S1. Arp5 knockout cells were successfully established, and they were confirmed to be devoid of Arp5 by Western blot analysis using an anti-Arp5 antibody.

\section{Western Blot Analysis}

Western blot analysis was performed as was described earlier (Kitayama et al., 2009) using anti-Arp5 (Kitayama et al., 2009), anti-Ino80 (Abcam), anti- $\alpha$-tubulin (Sigma), anti-MafK (Santa Cruz Biotechnology), anti-Bach1 (Dohi et al., 2008), or anti-Nrf2 (Santa Cruz Biotechnology) antibody. An anti-IgG conjugated to horseradish peroxidase (Promega) was used as the secondary antibody, and ECL Western blotting detection reagents (GE Healthcare) were used for the detection of bound antibodies.

\section{Quantitative RT-PCR Analysis}

Total RNA from human Nalm-6 pre-B cells was extracted with the RNeasy Mini kit (QIAGEN) following the manufacture's protocol. To prepare cDNA, an aliquot $(2.0 \mu \mathrm{g})$ of the extracted RNA was incubated with $10 \mu \mathrm{l}$ of 2 xMaster Mix (Applied Biosystems) (RT buffer, $100 \mathrm{mM}$ dNTP Mix, $10 \mathrm{mM}$ random primers, $1 \mu \mathrm{l}$ Multiscribe Reverse Transcriptase, RNase inhibitor, 
nuclease-free water) for $10 \mathrm{~min}$ at $25^{\circ} \mathrm{C}$, for $120 \mathrm{~min}$ at $37^{\circ} \mathrm{C}$ and finally for $5 \mathrm{~min}$ at $85^{\circ} \mathrm{C}$. An aliquot of this $\mathrm{cDNA}$ was used for quantitative PCR as described (Kusakabe et al., 2016). Quantitative PCR was carried out using gene specific primers for human HMOX1 (5' -CTCTCGAGCGTCCTCAGC- $3^{\prime}$ and $5^{\prime}$ TTCAGGGCCTCTGACAAATC- $\left.3^{\prime}\right)$ and for human $36 B 4\left(5^{\prime}-\right.$ CGACCTGGAAGTCCAACTAC- $3^{\prime}$ and $5^{\prime}$-ATCTGCTGCATC TGCTTG- $3^{\prime}$ ).

\section{Chromatin Immunoprecipitation (ChIP) Assay}

ChIP assays was performed as described earlier (Kimura et al., 2008) with some modifications. In brief, Nalm-6 pre-B cells (6$9 \times 10^{7}$ cells in a $100 \mathrm{ml}$ medium) were mixed with $2.7 \mathrm{ml}$ formaldehyde (the final concentration of $1 \%$ ) and shaken at room temperature for $5 \mathrm{~min}$ to crosslink proteins to DNA. To stop the crosslinking reaction, $17.5 \mathrm{ml}$ of $2 \mathrm{M}$ glycine was added, and the cells were first washed with $30 \mathrm{ml}$ PBS and then with $10 \mathrm{ml} \mathrm{NP}-40$ buffer [10 mM Tris- $\mathrm{HCl}$ ( $\mathrm{pH} 8.0$ ), $10 \mathrm{mM} \mathrm{NaCl}$ and $0.5 \% \mathrm{NP}-40$ ]. The cells were resuspended in $200 \mu \mathrm{l}$ SDS lysis buffer [ $50 \mathrm{mM}$ Tris- $\mathrm{HCl}(\mathrm{pH} 8.0), 10 \mathrm{mM}$ EDTA, and 1\% SDS], and to this $400 \mu \mathrm{l}$ ChIP dilution buffer [50 mM Tris-HCl (pH 8.0), $167 \mathrm{mM}$ $\mathrm{NaCl}, 1.1 \%$ Triton $\mathrm{X}-100$ and $0.11 \%$ sodium deoxycholate] was added. This mixture was sonicated using a Bioruptor (CosmoBio) for the shearing of the chromatin $(\sim 500 \mathrm{bp})$. After removing insoluble materials by centrifugation (12,000 g, $5 \mathrm{~min}$ ), $200 \mu \mathrm{l}$ ChIP dilution buffer was added to the supernatant to generate the input fraction for the ChIP analysis. Protein A- and GDynabeads (Invitrogen; $15 \mu$ l suspension of each) were washed and mixed with the ChIP input fraction and a specific antibody to the protein of interest. This mixture was incubated overnight at $4^{\circ} \mathrm{C}$ with rotation. Following the incubation, beads were washed sequentially with $1 \mathrm{ml}$ of RIPA [50 mM Tris- $\mathrm{HCl}$ (pH 8.0), $1 \mathrm{mM}$ EDTA, $0.1 \%$ SDS, $1 \%$ Triton X-100, 0.1\% sodium deoxycholate]$150 \mathrm{mM} \mathrm{NaCl}$ buffer, $1 \mathrm{ml}$ RIPA-500 mM NaCl buffer, and $1 \mathrm{ml}$ TE [10 mM Tris- $\mathrm{HCl}$ (pH 8.0) and $1 \mathrm{mM}$ EDTA]. After removing TE, beads were directly mixed with $200 \mu$ l elution buffer $[10 \mathrm{mM}$ Tris- $\mathrm{HCl}$ ( $\mathrm{pH}$ 8.0), $300 \mathrm{mM} \mathrm{NaCl}, 5 \mathrm{mM}$ EDTA, 0.5\% SDS] and incubated overnight at $65^{\circ} \mathrm{C}$ to reverse the cross-linking. Immunoprecipitated DNA was purified and analyzed by realtime PCR (ABI PRISM 7000 or CFX Connect Real-Time System). Obtained values were normalized with that of DNA prepared from the input fraction. Sequences of PCR primers used for the real-time PCR were: HO-1 E1 (5'-CAGTGCCTCCTCAGCTTC TC- $3^{\prime}$ and $5^{\prime}$-CTCGGTGGATTGCAACATTA-3'), HO-1 E2 (5' CTCTGCCCCTGCTGAGTAAT- $3^{\prime}$ and $5^{\prime}$-GAGCAGCTGGAA CTCTGAGG-3'), Rad54B intron9 (5'-TAGCTGGGACTGCAG GTGTA- $3^{\prime}$ and $5^{\prime}$-GTATTGCCAGGCCACAAGAT- $3^{\prime}$ ).

\section{Nuclease Accessibility Assay}

Nuclease accessibility assay was performed using the EpiQ chromatin analysis kit (BIO-RAD). Permeabilized Nalm-6 pre-B cells were treated with the nuclease supplied in the kit, the DNA was purified after the nuclease treatment, and the purified DNA was amplified using the region specific primers and reagents supplied in the EpiQ chromatin analysis kit according to the protocol provided by the manufacturer of the kit. Sequences of PCR primers for the E1, E2, exon5 of HMOX1 used in this assay were indicated above under ChIP Assay. Sequences of other primers used in this assay were: $\mathrm{HO}-1$ promoter $\left(5^{\prime}\right.$ GGGGGCTCTGGAAGGAGCAAAATCA-3' and $5^{\prime}$-CAGTGT GGGGTGGAGAGGAGCAGTC-3'), GAPDH promoter: (5' CGCACGTAGCTCAGGCCTCAAGACC- $3^{\prime}$ and $5^{\prime}$-GGCTGA CTGTCGAACAGGAGGAGCA-3').

\section{RESULTS}

\section{Establishment of an Arp5 Gene Knockout Human Cell Line}

We generated human Arp5 KO cells and utilized them to analyze the role of Arp5 in the INO80 chromatin remodeling complex. Both the alleles of the ARP5 gene in the human Nalm- 6 pre-B cell line were disrupted using conventional gene knockout methods and an Arp5 KO cell line was established (Supplementary Figure S1). Western blot analysis by using a specific anti-Arp5 antibody confirmed that no Arp5 was expressed in Arp5 KO cells (Supplementary Figure S2A). Knockout of ARP5 gene did not bestow lethal phenotype in Arp5 KO cells; however, the growth of Arp5 KO cells was slightly slower than that of the wild-type cells (Supplementary Figure S2B). These Arp5 KO cells, along with Arp8 KO cells (Osakabe et al., 2014), were used for further analyzing the function of INO80 complex.

\section{INO80 Complex Is Involved in Oxidative Stress-Induced Expression of HMOX1}

To determine whether the INO80 complex is involved in transcriptional regulation of $H M O X 1$, we analyzed the expression levels of HMOX1 mRNA in wild-type, Arp5 KO, and Arp8 KO cells. The expression level of HMOX1 was moderately decreased both in Arp5 $\mathrm{KO}$ and Arp8 $\mathrm{KO}$ cells in the absence of oxidative stress with hemin (Figure 1, left panel). However, significantly decreased expression levels of HMOX1 were observed in both $\mathrm{KO}$ cells, as compared to that in the wild-type cells, following induction with oxidative stress; notably, the expression level of HMOX1 in Arp5 KO and Arp8 KO cells was $~ 1 / 100$ of that of the wild-type cells (Figure 1, right panel). This result suggested that the INO80 complex is required for the induction of HMOX1 expression with hemin and that Arp5 and Arp8 may have essential roles in INO80complex function.

We also performed a genome-wide microarray transcription analysis of control (without oxidative stress) and oxidative stressinduced Arp5-KO and Arp8-KO cells. The relative change in transcription of each gene in the gene loci was calculated by comparing its transcription level in Arp5 $\mathrm{KO}$ or Arp8 $\mathrm{KO}$ cells with that in the wild-type cells. The raw data and experimental details were deposited in the GEO database under accession number GSE66888. We plotted the degree of change in the transcription level of each gene whose transcription was misregulated in Arp5 $\mathrm{KO}$ or Arp8 KO cells (>2-fold) (Figure 2A). The genes belonging to the groups I to IV under each condition are listed in Supplementary Tables S1-S8. While analyzing the results obtained from the cells without oxidative stress (Figure 2A, left panel) and cells induced with oxidative 

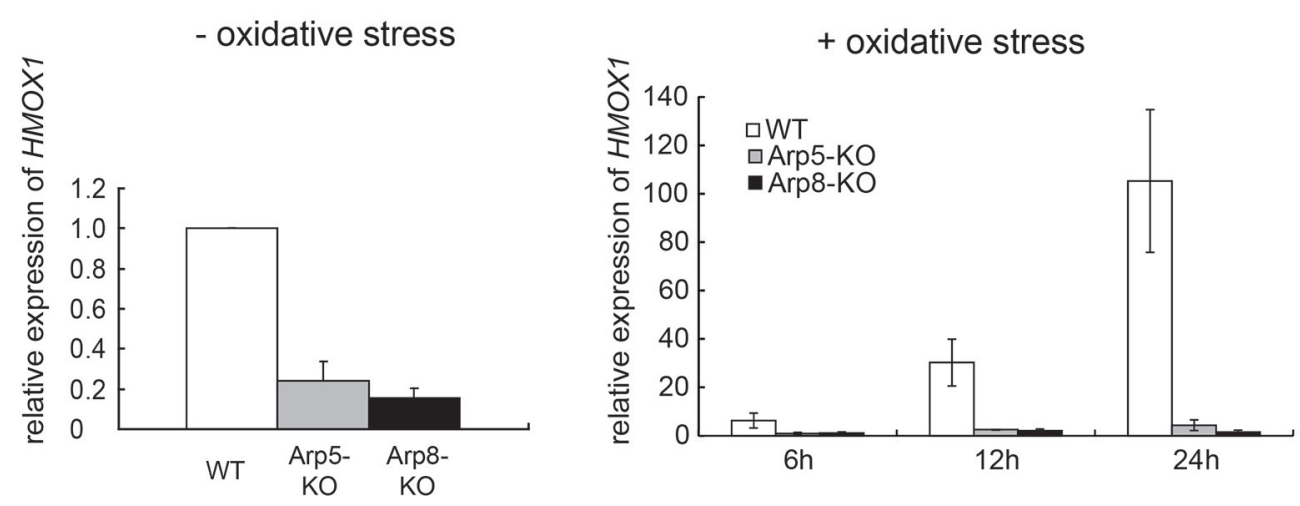

FIGURE 1 | The INO80 complex is involved in HMOX1 expression. Quantitative RT-PCR (qRT-PCR) analysis of HMOX1 mRNA in wild-type (WT), Arp5 KO, and Arp8 KO cells. Left panel: control cells (not treated with hemin) and Right panel: cells treated with $20 \mu \mathrm{M}$ hemin, an oxidative stress inducer, for the indicated time. The expression level of HMOX1 in wild-type control cells (without oxidative stress) was arbitrarily set at 1 . The expression of $36 \mathrm{~B} 4$ gene was used to normalize the results. Averages of at least three independent experiments ( \pm standard deviation) are shown.

\section{A}

- oxidative stress

+ oxidative stress
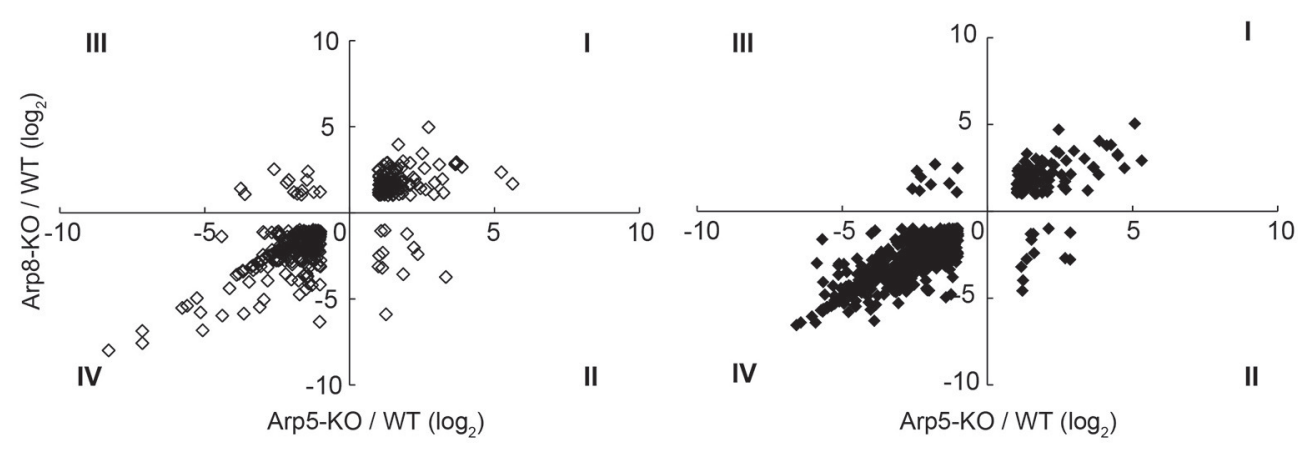

B

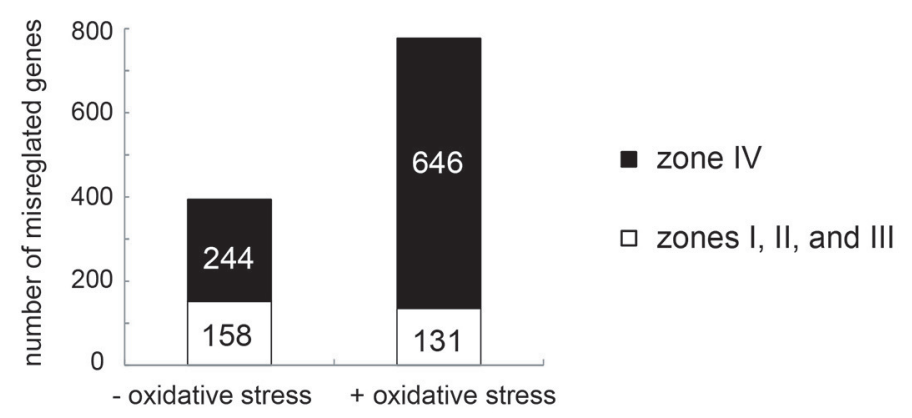

FIGURE 2 | Microarray analysis of Arp5 KO and Arp8 KO cells induced with or without oxidative stress. (A) Genes whose transcriptional changes were significant in Arp5 KO and Arp8 KO cells under control (without oxidative stress, left panel) and experimental (with oxidative stress, right panel) conditions were plotted according to their $\log _{2}$ ratios. (B) Zone plot of transcript expression. Genes which were misregulated in these KO cells were counted and number of genes expressed in each zone, as shown in (A) above, were plotted. The filled bar indicates the number of genes in zone IV, expression levels of which were down-regulated both in Arp5 KO and Arp8 KO cells. The open bar indicates total number of other misregulated genes (Zones I, II, and III) in Arp5-KO and Arp8-KO cells.

stress (Figure 2A, right panel), we found a correlation between the degree of misregulation observed in Arp5 KO (horizontal axis) and Arp8 KO (vertical axis) cells $\left(R^{2}=0.64\right.$ and 0.77 in the absence and presence of oxidative stress, respectively); more than $90 \%$ of the misregulated genes were found either in zone I (upregulated in both cell types) or in zone IV (downregulated in both cell types) in the zone plot of transcript expression (Figure 2B). This observation suggested that both Arp5 and 
Arp 8 are required for the transcriptional regulation mediated by the INO80 complex. Interestingly, the number of genes found in the zone IV of the zone plot were significantly increased following the induction with oxidative stress (Figure 2B, $p=1.9$ $\left.\times 10^{-17}\right)$. In the absence of oxidative stress, genes belonging to eight GO categories are significantly accumulated $(p<0.01)$ in the zone IV (Supplementary Table S9). On the other hand, in the presence of oxidative stress, genes belonging to $48 \mathrm{GO}$ categories are significantly accumulated in the zone IV (Supplementary Table S10). Genes for heme oxygenase 1 (HMOX1), glutathione peroxidase (GPX3), and glutathione S-transferase (GSTM3) are included in the zone IV only in the presence of oxidative stress. These observations suggest that the INO80 complex is involved in the expression of not only typical oxidative stress genes but also many other genes.

\section{Arp8, but Not Arp5, Is Involved in the Binding of Ino80 to Chromatin}

Human Arp8 has been shown to have histone- and DNA-binding activities, which were suggested to contribute to the DSB repair function of the INO80 complex (Kashiwaba et al., 2010; Osakabe et al., 2014; Gerhold et al., 2015). Here, we used Arp8 KO cells to examine whether Arp8 is involved in the binding of INO80 complex to chromatin without inducing DNA damage. When we analyzed the amount of Ino80 in the chromatin fraction prepared from the wild-type, Arp5 KO, and Arp8 KO cells by Western blotting, we observed that the amount of chromatin-bound Ino80 in Arp8 KO cells decreased to around $50 \%$ of that in the wildtype cells (Figure 3, Arp8-KO). On the other hand, absence of Arp5 (Arp5 KO cells) did not have any significant effect on the binding of Ino80 to chromatin (Figure 3, Arp5-KO). These results suggested that Arp8, but not Arp5, contributes to the chromatin binding property of the INO80 complex.

\section{INO80 Complex Binds to the Regulatory Sites of HMOX1}

Induction of HMOX1 expression is under the control of Mafrecognition elements (MARE). Two enhancer regions, one distal
(E2) and another proximal (E1), have been identified upstream of the HO-1 coding region (Figure 4A); sequences of both enhancers conform to the sequence of the MARE (Igarashi and Watanabe-Matsui, 2014). To elucidate the role of Arp5 in the activation of HMOX1 by the INO80 complex, we performed chromatin immunoprecipitation (ChIP) assays using anti-Arp5 (Figure 4B, upper-left panel), anti-Arp8 (Figure 4B, upper-right panel), and anti-Ino80 (Figure 4B, lower-right panel) antibodies. As shown in Figure $4 B$, in the absence of oxidative stress (open bars), Arp5, Arp8, and Ino80 exhibited slight, but significant binding to the E1 and E2 sites as compared to an INO80-free Rad54B site (Park et al., 2010). The bindings of Arp5 to the E1 and E2 sites in the absence of oxidative stress were confirmed by the comparison of ChIP results with anti-Arp5 and control antibodies (Supplementary Figure S3). After inducing oxidative stress, the bindings of Arp5, Arp8, and Ino80 to the E1 and E2 sites were increased (Figure $\mathbf{4 B}$, filled bars). These results suggested binding of the INO80 complex to the regulatory sites of HMOX 1 in response to oxidative stress.

\section{Arp5 Is Not Required for the Binding of Ino80 to Chromatin}

To test whether Arp5 is required for the binding of INO80 complex to the regulatory sites of HMOX1, ChIP assay was performed using wild-type and Arp5 KO cells and an anti-Ino80 antibody. Interestingly, the binding of Ino80 to the E1 and E2 sites were not impaired in Arp5 KO cells both in the absence and presence of oxidative stress (Figure 4C, left and right panels, respectively). This result suggested that Arp5 is not required for the binding of INO80 complex to the E1 and E2 sites regardless of whether or not the cells were subjected to oxidative stress.

\section{Arp5 Is Required for the Chromatin Remodeling at the Regulatory Sites of HMOX1}

To test the possibility whether Arp5 is required for the remodeling of chromatin by the INO80 complex, we analyzed the structure of chromatin at the E2 and E1 sites of HMOX1.
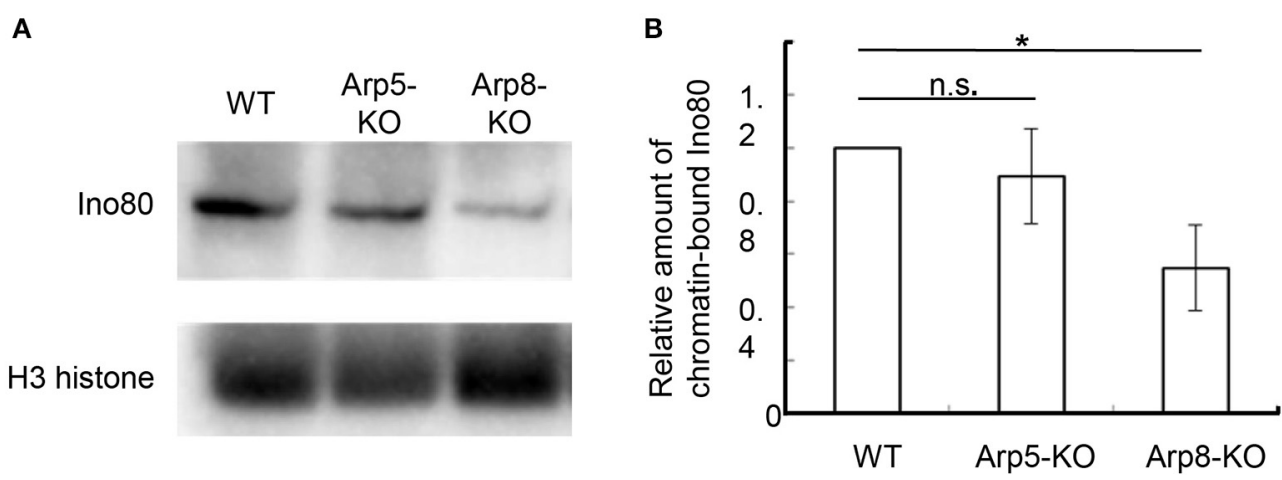

FIGURE 3 | Quantification of chromatin-bound Ino80 in wild-type, Arp5 KO, and Arp8 KO cells. (A) The chromatin fractions prepared from the indicated cells were immunoblotted with an anti-Ino80 antibody (top panel) and an anti-H3 antibody (lower panel), which was used as an internal control. (B) The intensity of the chromatin-bound Ino80 band was normalized with respect to that of H3 band in each case, and the relative amount of chromatin-bound Ino80 in each cell line was normalized with respect to that in the wild-type cells. Student's $t$-test was used to determine the $P$-value. ${ }^{*} P<0.05$. 
A

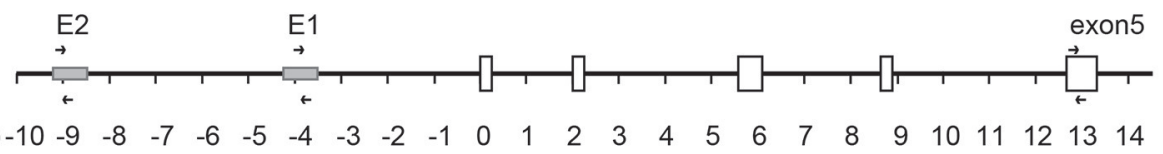

B

Arp5 biniding

Arp8 biniding
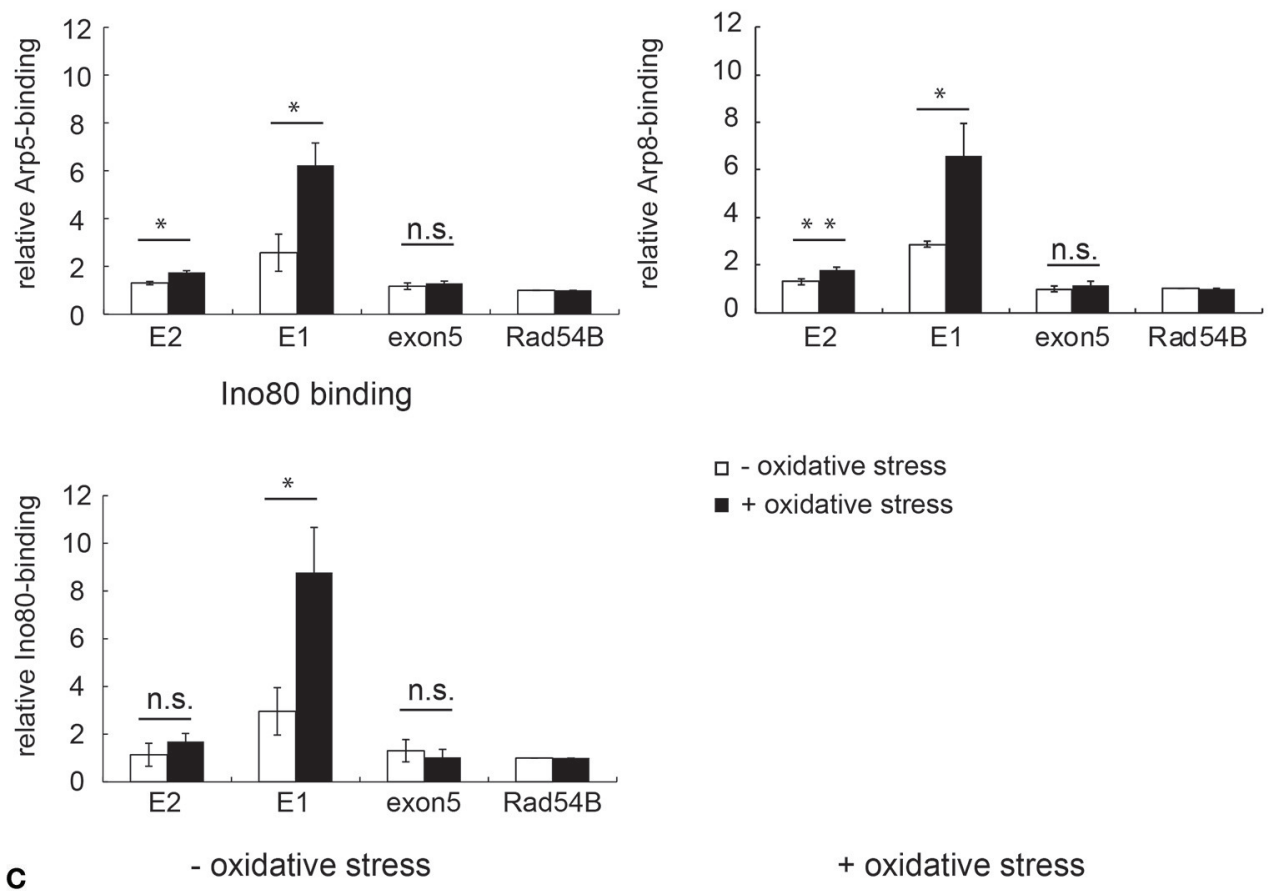

$\square$ - oxidative stress

- + oxidative stress
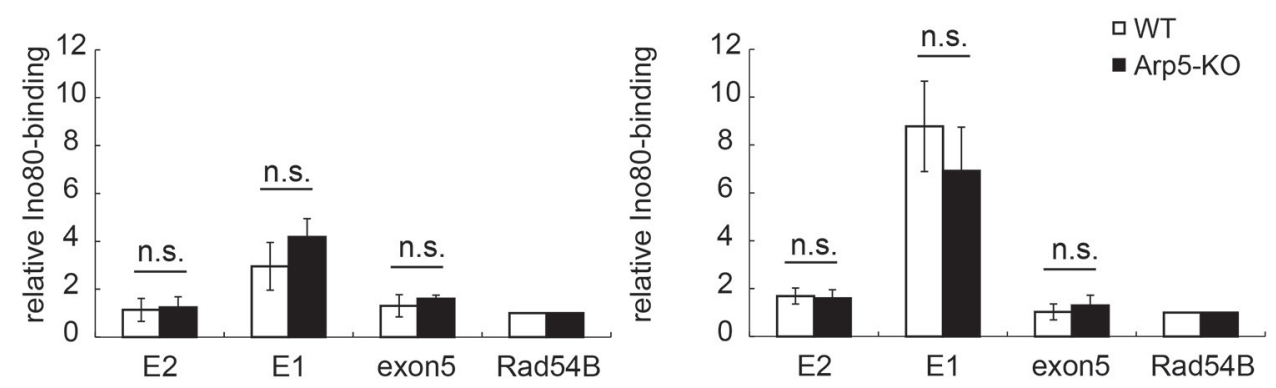

FIGURE 4 | Analysis of the binding of INO80 complex to the regulatory sites of HMOX1. (A) Schematic representation of the human HMOX1 locus. Arrows indicate positions of PCR primer pairs used for the ChIP analysis. (B) The quantitative-ChIP assay was performed by using anti-Arp5 (B, upper-left panel), anti-Arp8 (B, upper-right panel), and anti-Ino80 (B, lower-right panel) antibodies. The amount of immunoprecipitated fragment for each site was normalized with respect to the input fraction value, and was shown as relative enrichment to that of the INO80-free Rad54 site (Park et al., 2010). Open and filled bars represent chromatin binding in control (without oxidative stress) and experimental (with oxidative stress) cells, respectively. (C) The binding of Ino80 to the regulatory sites in wild-type (WT) and Arp5-KO cells was analyzed by ChIP assay by using an anti-Ino80 antibody in control (without oxidative stress, left panel) and experimental (with oxidative stress, right panel) cells. Open and filled bars represent chromatin-bound Ino80 in WT and Arp5-KO cells, respectively. Data shown are averages from at least three independent experiments ( \pm standard deviation). ${ }^{*} P<0.05$ (in $\mathbf{B}, \mathbf{C}$ ).

Since open form of chromatin permits accessibility of chromatin DNA to exogenous nuclease digestion, we determined nuclease accessibility of chromatin at the regulatory sites of the wild-type and Arp5 KO cells (Figure 5, left and right panels, respectively) by quantifying the un-cut DNA by qPCR. Under oxidative stress condition, chromatin accessibility to nuclease at the E2 and E1 sites and also at a promoter site adjacent to the E1 site increased similarly in the wild-type cells (Figure 5, left panel). On the other hand, although chromatin accessibility to nuclease at the E2 and the promoter sites did not change upon induction with oxidative 
WT

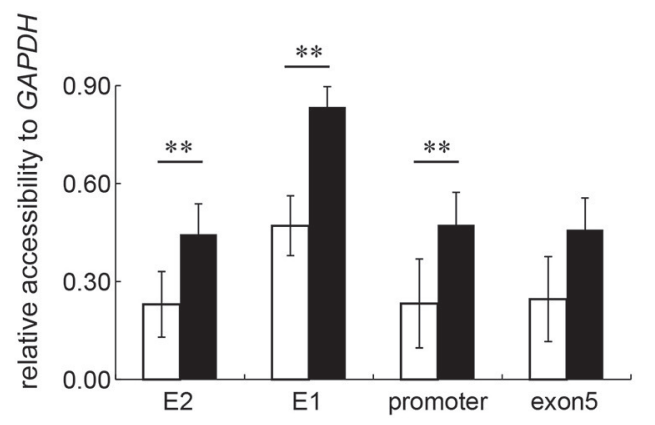

Arp5 KO

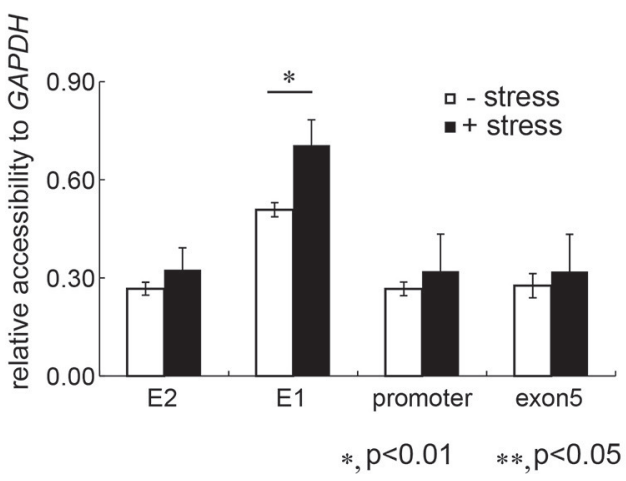

FIGURE 5 | Comparison of chromatin structure at the regulatory element sites of $\boldsymbol{H M O X 1 . ~ C h r o m a t i n ~ i s ~ i s o l a t e d ~ f r o m ~ w i l d - t y p e ~ ( W T ) ~ a n d ~ A r p 5 ~ d e f i c i e n t ~ c e l l s , ~}$ treated with nuclease, genomic DNA is then isolated and chromatin accessibility to nucleases was measured by amplifying the purified DNA using quantitative-PCR. If the chromatin forms a loose structure, the PCR amplification efficiency is low because the genomic DNA is digested by the nuclease. The nuclease accessibility value obtained for each regulatory site of HMOX1 was normalized with that for the GAPDH promoter site, and was shown relative to the nuclease accessibility value obtained for the exon 5 site in control cells (without oxidative stress). Data shown are averages from at least three independent experiments ( \pm standard deviation). ${ }^{\star} P<0.05 ;{ }^{\star \star} P<0.01$.

stress, the increase in accessibility at the E1 site was partially impaired when Arp5 KO cells were subjected to oxidative stress (Figure 5, right panel). These results suggested that, in response to oxidative stress, the INO80 complex remodels chromatin structure at the regulatory regions of HMOX1 and that Arp5 is required for this activity of the INO80 complex. At the E1 site, some additional chromatin remodeling complexes might be involved in the change of chromatin structure.

\section{Arp5 Is Required for Binding of Chromatin to the HMOX1 Activator}

In the transcriptional regulation of $H M O X 1$, a small Maf oncoprotein, MafK, directly binds to MARE sequences at the E1 and E2 sites of $H M O X 1$, and regulates both repression and activation of HMOX 1 expression depending on its dimeric partner (Igarashi and Watanabe-Matsui, 2014). In the absence of oxidative stress, the repressor Bach1 associates with the E1 and E2 sites by forming a heterodimer with MafK and represses HMOX1. On the other hand, under oxidative stress, Bach1 is released from the chromatin when the NF-E2-related factor 1 (Nrf2) associates with the E1 and E2 sites by forming a heterodimer with MafK, which remains on the chromatin, and activates HMOX1 (Figure 6A). To examine the involvement of Arp5 in the association and/or dissociation processes of these transcription factors in the induced activation of HMOX1 by oxidative stress, ChIP assays were carried out using the wild-type and Arp5 KO cells and specific antibodies against these transcription factors. Both in wild-type and Arp5 KO cells, consistently similar level of MafK binding was observed at the E1 and E2 sites under both control (no oxidative stress) and oxidative stress conditions (Figure 6B).

In wild-type cells, as was reported previously (Igarashi and Watanabe-Matsui, 2014), the association of Bach1 to the E1 and E2 sites was observed under no oxidative stress condition (Figure 6C, left panel), but not under oxidative stress condition (Figure 6C, right panel). In Arp5-KO cells, the association and dissociation of Bach1 were not disturbed (Figure 6C, Arp5-KO).

The binding of Nrf2 to E2 and E1 sites increased in response to oxidative stress in wild-type cells (Figure 6D, WT), as was reported previously (Igarashi and Watanabe-Matsui, 2014). In contrast, the binding of $\mathrm{Nrf} 2$ to $\mathrm{E} 2$ and $\mathrm{E} 1$ sites in response to oxidative stress was significantly impaired in Arp5-KO cells (Figure 6D, Arp5-KO). However, the total amount of Nrf2 and Mafk proteins in the Arp5 KO cells was similar to that in the wild-type cells (Supplementary Figure S4). Taken together with the observations that Arp5 binds to the E1 and E2 sites even in the absence of oxidative stress and that its binding to these sites was increased in response to the stress, these results tend to suggest that Arp5-dependent remodeling of chromatin by the INO80 complex is required for the binding of $\mathrm{Nrf} 2$ to the regulatory sites of HMOX1.

\section{DISCUSSION}

\section{Roles of Actin Family Proteins in the INO80 Complex}

By using Arp5 $\mathrm{KO}$ and Arp8 $\mathrm{KO}$ cells, we have been able to successfully demonstrate here that Arp5 and Arp8 have roles in the INO80 chromatin remodeling complex, contributing to the induction of HMOX1 by oxidative stress. We previously reported that the conditional $\mathrm{KO}$ of Arp8 leads to cell lethality (Osakabe et al., 2014). In the present study, we demonstrated that $\mathrm{KO}$ of Arp5 causes cells to grow slow, but does not cause cell lethality (Supplementary Figure S2). These observed differences in phenotypes of Arp5 KO and Arp8 KO cells support the contention that the actin family 
A
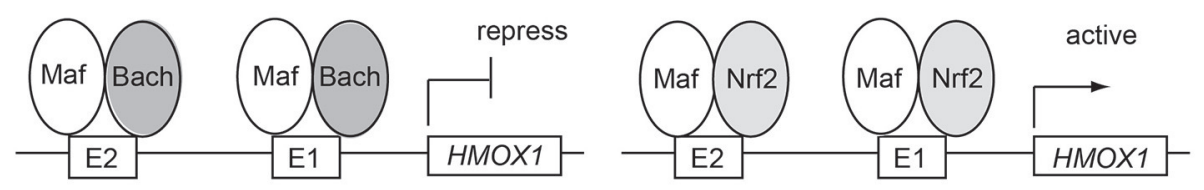

B - oxidative stress

+ oxidative stress
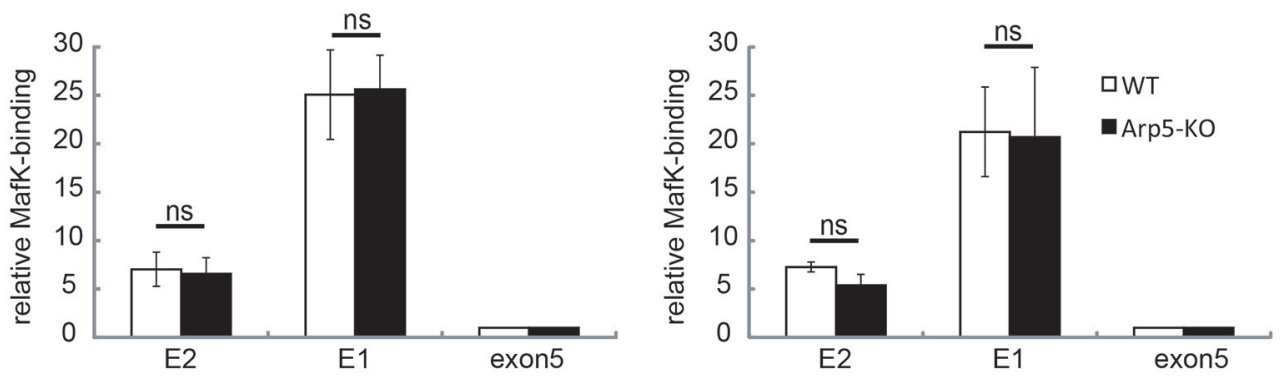

C

- oxidative stress

+ oxidative stress
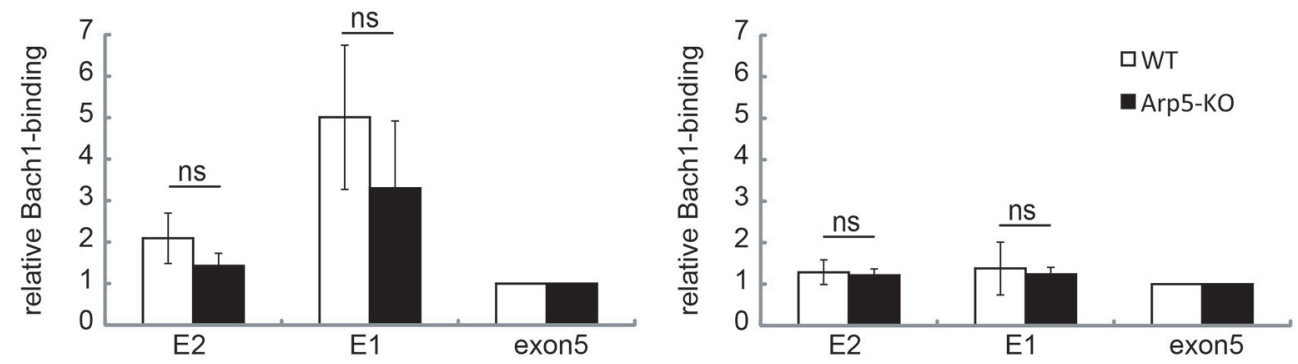

D

- oxidative stress
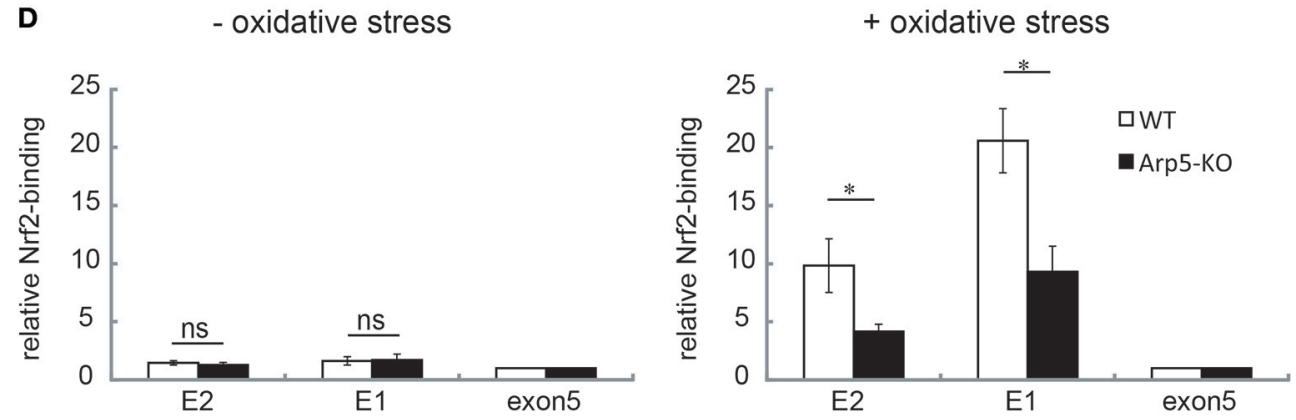

FIGURE 6 | Analysis of the binding of transcription regulators to the E2 and E1 sites of HMOX1 in wild-type and Arp5-KO cells. (A) In the absence of any oxidative stress (left panel), the repressor Bach1 forms a heterodimer with MafK, which binds to the Maf recognition element (MARE) at the E2 and E1 sites, and represses HMOX1 transcription. In the presence of oxidative stress (right panel), Bach1 is released, and the activator Nrf2 forms a heterodimer with MafK and binds to the MARE elements at E2 and E1. To analyze the binding of these transcriptional regulators, quantitative-ChIP assay was performed using antibodies against MafK (B), Bach1 (C), and Nrf2 (D) in wild-type (open bar) and Arp5-KO (filled bar) cells. The amount of immunoprecipitated fragment was normalized with respect to the input fraction value. Data shown are binding relative to that of exon 5 . Averages from at least three independent experiments ( \pm standard deviation) are shown. $P$-value (Student's $t$-test) for the difference between WT and Arp5 KO cells is indicated. ${ }^{*} P<0.05$.

proteins Arp5 and Arp8 have distinctive roles in the INO80 complex.

Although the structural details of the human INO80 complex are not known yet, the fact that the components of the yeast and human INO80 complexes are evolutionarily conserved implies a high degree of structural similarity between these complexes (Gerhold and Gasser, 2014). Arp8, but not Arp5, is included in the Arp8 module together with actin and Arp4
(Tosi et al., 2013). The Arp8 module directly associates with the helicase-SANT-associated (HSA) domain of the enzymatic Ino80 ATPase scaffold. Arp8 has both histone- and DNAbinding activities (Gerhold et al., 2012; Osakabe et al., 2014), and Arp4 also has histone binding activity (Harata et al., 1999; Nishimoto et al., 2012). Although actin itself has neither histone- nor DNA-binding activity, actin in the budding yeast INO80 complex is required for the binding of the INO80 
complex to extranucleosomal DNA (Bartholomew, 2013; Kapoor et al., 2013). Consistent with this observation, a subcomplex comprising of the actin family molecules belonging to the Arp8 subdomain (actin, Arp4, and Arp8) and the HSA domain fragment of Ino80 exhibited significantly more affinity for DNA binding than expected from the individual subunits (Gerhold et al., 2012). These observations suggested that, despite displaying different characteristics, actin family proteins in the Arp8 module (i.e., actin, Arp4, and Arp8) function in a cooperative manner so that the INO80 complex could bind to chromatin.

Arp5 is also shown to be required for the function of the INO80 complex in budding yeast (Yao et al., 2015, 2016). Arp5 is included in the Arp5 module, which indirectly associate with the enzymatic Ino80 ATPaes scaffold through the Rvb module (Tosi et al., 2013). In addition, Arp5 by itself, unlike Arp8, is not able to bind to either DNA or histone. The distinctive functions of Arp5 and Arp8 in the INO80 complex may rise from their topological distribution on the INO80 complex and differences in their biochemical characters.

\section{A Model Depicting the Function of Arp5 and Arp8 in HMOX1 Expression}

Based on our observations, together with the results described in previous reports (Zhang et al., 2006; Maruyama et al., 2013; Igarashi and Watanabe-Matsui, 2014), we propose a model depicting the roles Arp5 and Arp8 might play in the oxidative stress-induced activation of HMOX1 (Figure 7). In the proposed model, the INO80 complex initially binds to the regulatory sites of HMOX1 with the help of Arp8, but not that of Arp5, in response to oxidative stress. The repressor Bach1 is released from the regulatory sites independently of the activity of the INO80 complex (top). Next, the chromatin remodeling activity of the INO80 complex is induced with the help of Arp5. The remodeled chromatin structure induces the biding of the activator Nrf2 to the regulatory sites (middle). Finally, another chromatin remodeling complex BRG1 has been reported to be recruited to these sites in an Nrf2-dependent manner (Zhang et al., 2006; Maruyama et al., 2013). The BRG1 complex is shown to alter the B-DNA structure at the HMOX1 promoter to Z-DNA structure (Maruyama et al., 2013; Zhang et al., 2006). Z-DNA formation reduces nucleosome occupancy and induces RNA polymerase II recruitment to the $H M O X 1$ promoter, and thereby activates the transcription of HMOX1 (bottom).

\section{Involvement of Nuclear Actin Family Proteins in the Maintenance of Genome Integrity}

The oxidative stress causes injury to genome DNA, and this leads to carcinogenesis, Alzheimer, aging, and various other diseases (Ryter et al., 2006; Klaunig et al., 2010; Coppedè and Migliore, 2015; Foppoli et al., 2015; Loboda et al., 2016). Oxidative stress inducible genes help in protecting the genome from the stress. For example, HO-1 has a strong reducing capacity and removes substances responsible for ROS formation. Therefore,

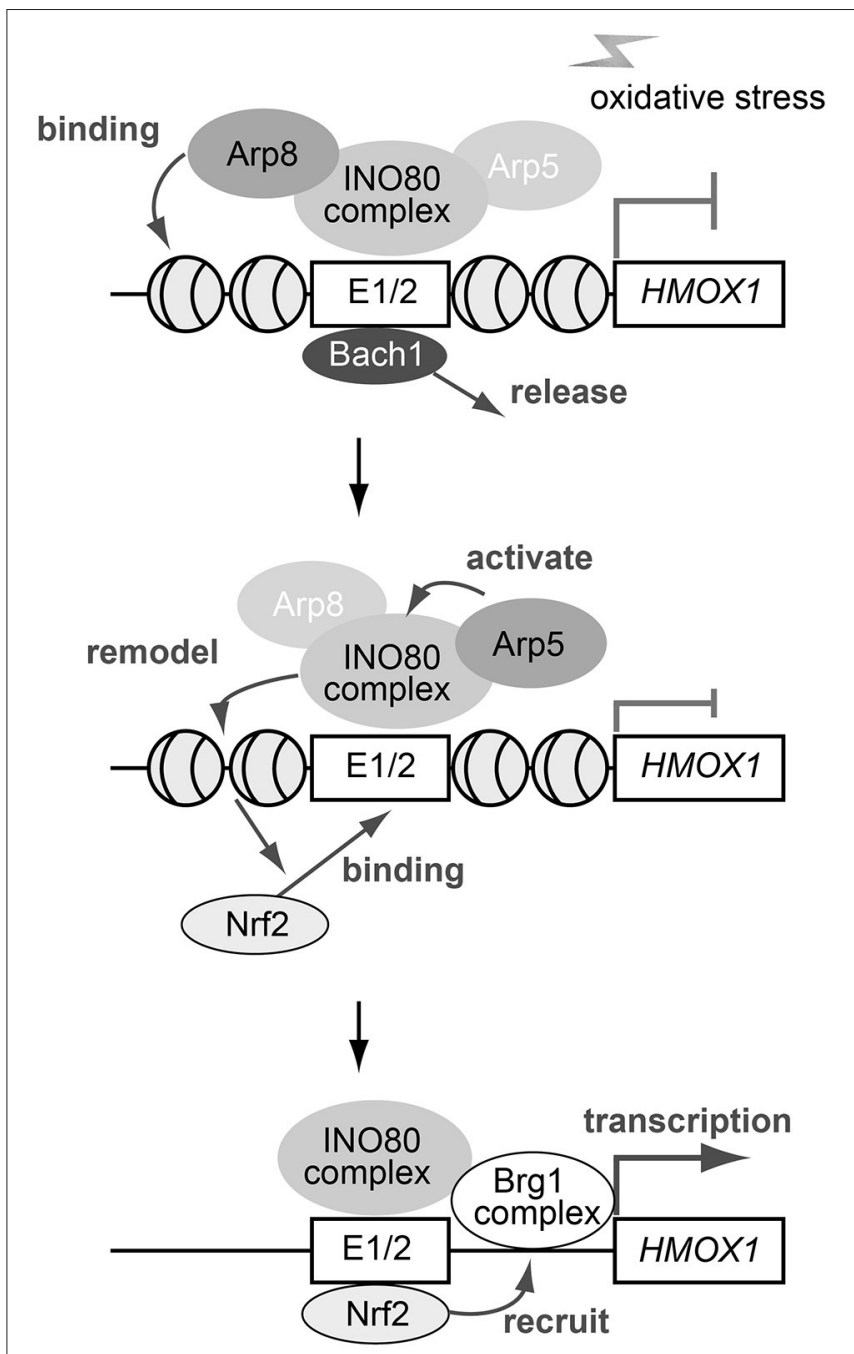

FIGURE 7 | A schematic model depicting the roles of Arp5 and Arp8 in the oxidative stress-induced expression of HMOX1. See text for details.

the INO80 complex appears to play a role in the maintenance of genome integrity by activating oxidative stress inducible genes including HMOX1. It is also known that the INO80 complex helps maintaining the genome stability via its roles in DNA replication and DSB repair (Kawashima et al., 2007; Shimada et al., 2008; Kashiwaba et al., 2010; Gerhold et al., 2015). Based on the known broad functions of the INO80 complex in stress response and DNA metabolism, it could be conferred that Arp5 and Arp8, possibly together with actin and Arp4, may have indispensable roles in the maintenance of genome integrity.

\section{AUTHOR CONTRIBUTIONS}

YT, HM, YA, YK, YO, HN, and KS contributed to the acquisition, analysis, and interpretation of data. $\mathrm{KI}$ and $\mathrm{MH}$ made substantial contribution to the conception and design of the work. All authors participated in drafting the manuscript, approval for 
publication, and agree to be accountable for all aspects of this work.

\section{ACKNOWLEDGMENTS}

We thank Drs. Atsushi Maruyama and Ken Ito for providing some of the materials used in this study. This work was supported by JSPS KAKENHI Grant Number JP25116009 and

\section{REFERENCES}

Bartholomew, B. (2013). Monomeric actin required for INO80 remodeling. Nat. Struct. Mol. Biol. 20, 405-407. doi: 10.1038/nsmb.2553

Cai, Y., Jin, J., Yao, T., Gottschalk, A. J., Swanson, S. K., Wu, S., et al. (2007). YY1 functions with INO80 to activate transcription. Nat. Struct. Mol. Biol. 14, 872-874. doi: 10.1038/nsmb1276

Camhi, S. L., Alam, J., Otterbein, L., Sylvester, S. L., and Choi, A. M. (1995). Induction of heme oxygenase-1 gene expression by lipopolysaccharide is mediated by AP-1 activation. Am. J. Respir. Cell Mol. Biol. 13, 387-398. doi: 10.1165/ajrcmb.13.4.7546768

Coppedè, F., and Migliore, L. (2015). DNA damage in neurodegenerative diseases. Mutat. Res. 776, 84-97. doi: 10.1016/j.mrfmmm.2014.11.010

Dion, V., Shimada, K., and Gasser, S. M. (2010). Actin-related proteins in the nucleus: life beyond chromatin remodelers. Curr. Opin. Cell Biol. 22, 383-391. doi: 10.1016/j.ceb.2010.02.006

Dohi, Y., Ikura, T., Hoshikawa, Y., Katoh, Y., Ota, K., Nakanome, A., et al. (2008). Bach1 inhibits oxidative stress-induced cellular senescence by impeding p53 function on chromatin. Nat. Struct. Mol. Biol. 15, 1246-1254. doi: $10.1038 / \mathrm{nsmb} .1516$

Fenn, S., Breitsprecher, D., Gerhold, C. B., Witte, G., Faix, J., and Hopfner, K. P. (2011). Structural biochemistry of nuclear actin-related proteins 4 and 8 reveals their interaction with actin. EMBO J. 30, 2153-2166. doi: 10.1038/emboj.2011.118

Foppoli, C., De Marco, F., Cini, C., and Perluigi, M. (2015). Redox control of viral carcinogenesis: the human papillomavirus paradigm. Biochim. Biophys. Acta 1850, 1622-1632. doi: 10.1016/j.bbagen.2014.12.016

Gerhold, C. B., and Gasser, S. M. (2014). INO80 and SWR complexes: relating structure to function in chromatin remodeling. Trends Cell. Biol. 24, 619-631. doi: 10.1016/j.tcb.2014.06.004

Gerhold, C. B., Hauer, M. H., and Gasser, S. M. (2015). INO80-C and SWR-C: guardians of the genome. J. Mol. Biol. 427, 637-651. doi: 10.1016/j.jmb.2014.10.015

Gerhold, C. B., Winkler, D. D., Lakomek, K., Seifert, F. U., Fenn, S., Kessler, B., et al. (2012). Structure of Actin-related protein 8 and its contribution to nucleosome binding. Nucleic Acids Res. 40, 11036-11046. doi: 10.1093/nar/gks842

Harata, M., Oma, Y., Mizuno, S., Jiang, Y. W., Stillman, D. J., and Wintersberger, U. (1999). The nuclear actin-related protein of Saccharomyces cerevisiae, Act3p/Arp4, interacts with core histones. Mol. Biol. Cell 10, 2595-2605. doi: $10.1091 / \mathrm{mbc}$.10.8.2595

Igarashi, K., and Watanabe-Matsui, M. (2014). Wearing red for signaling: the heme-bach axis in heme metabolism, oxidative stress response and iron immunology. Tohoku J. Exp. Med. 232, 229-253. doi: 10.1620/tjem. 232.229

Kapoor, P., Chen, M., Winkler, D. D., Luger, K., and Shen, X. (2013). Evidence for monomeric actin function in INO80 chromatin remodeling. Nat. Struct. Mol. Biol. 20, 426-432. doi: 10.1038/nsmb.2529

Kashiwaba, S., Kitahashi, K., Watanabe, T., Onoda, F., Ohtsu, M., and Murakami, Y. (2010). The mammalian INO80 complex is recruited to DNA damage sites in an ARP8 dependent manner. Biochem. Biophys. Res. Commun. 402, 619-625. doi: 10.1016/j.bbrc.2010.10.066

Katoh, Y., Ikura, T., Hoshikawa, Y., Tashiro, S., Ito, T., Ohta, M., et al. (2011). Methionine adenosyltransferase II serves as a transcriptional corepressor of Maf oncoprotein. Mol. Cell 41, 554-566. doi: 10.1016/j.molcel.2011.02.018
JP15H013400 to MH, JP15H02506 to KI, and the Human Frontier Science Program (RGP0017) to MH.

\section{SUPPLEMENTARY MATERIAL}

The Supplementary Material for this article can be found online at: http://journal.frontiersin.org/article/10.3389/fgene. 2017.00017/full\#supplementary-material

Kawashima, S., Ogiwara, H., Tada, S., Harata, M., Wintersberger, U., Enomoto, T., et al. (2007). The INO80 complex is required for damageinduced recombination. Biochem. Biophys. Res. Commun. 355, 835-841. doi: 10.1016/j.bbrc.2007.02.036

Kimura, H., Hayashi-Takanaka, Y., Goto, Y., Takizawa, N., and Nozaki, N. (2008). The organization of histone $\mathrm{H} 3$ modifications as revealed by a panel of specific monoclonal antibodies. Cell Struct. Funct. 33, 61-73. doi: 10.1247/csf. 07035

Kitamura, H., Matsumori, H., Kalendova, A., Hozak, P., Goldberg, I. G., Nakao, M., et al. (2015). The actin family protein ARP6 contributes to the structure and the function of the nucleolus. Biochem. Biophys. Res. Commun. 464, 554-560. doi: 10.1016/j.bbrc.2015.07.005

Kitayama, K., Kamo, M., Oma, Y., Matsuda, R., Uchida, T., Ikura, T., et al. (2009). The human actin-related protein hArp5: nucleo-cytoplasmic shuttling and involvement in DNA repair. Exp. Cell Res. 315, 206-217. doi: 10.1016/j.yexcr.2008.10.028

Klaunig, J. E., Kamendulis, L. M., and Hocevar, B. A. (2010). Oxidative stress and oxidative damage in carcinogenesis. Toxicol. Pathol. 38, 96-109. doi: $10.1177 / 0192623309356453$

Kusakabe, M., Oku, H., Matsuda, R., Hori, T., Muto, A., Igarashi, K., et al. (2016). Genetic complementation analysis showed distinct contributions of the Nterminal tail of H2A.Z to epigenetic regulations. Genes Cells 21, 122-135. doi: $10.1111 /$ gtc. 12327

Loboda, A., Damulewicz, M., Pyza, E., Jozkowicz, A., and Dulak, J. (2016). Role of Nrf2/HO-1 system in development, oxidative stress response and diseases: an evolutionarily conserved mechanism. Cell. Mol. Life Sci. 73, 3221-3247. doi: 10.1007/s00018-016-2223-0

Maeshima, H., Sato, M., Ishikawa, K., Katagata, Y., and Yoshida, T. (1996). Participation of altered upstream stimulatory factor in the induction of rat heme oxygenase-1 by cadmium. Nucleic Acids Res. 24, 2959-2965. doi: 10.1093/nar/24.15.2959

Maruyama, A., Mimura, J., Harada, N., and Itoh, K. (2013). Nrf2 activation is associated with Z-DNA formation in the human HO-1 promoter. Nucleic Acids Res. 41, 5223-5234. doi: 10.1093/nar/gkt243

Nishimoto, N., Watanabe, M., Watanabe, S., Sugimoto, N., Yugawa, T., Ikura, T., et al. (2012). Heterocomplex formation by Arp4 and beta-actin is involved in the integrity of the Brg1 chromatin remodeling complex. J. Cell Sci. 125, 3870-3882. doi: 10.1242/jcs.104349

Oma, Y., and Harata, M. (2011). Actin-related proteins localized in the nucleus: from discovery to novel roles in nuclear organization. Nucleus 2, 38-46. doi: $10.4161 /$ nucl.14510

Ono, T., Nishijima, H., Adachi, N., Iiizumi, S., Morohoshi, A., Koyama, H., et al. (2009). Generation of tetracycline-inducible conditional gene knockout cells in a human Nalm-6 cell line. J. Biotechnol. 141, 1-7. doi: 10.1016/j.jbiotec.2008.12.010

Osakabe, A., Takahashi, Y., Murakami, H., Otawa, K., Tachiwana, H., Oma, Y., et al. (2014). DNA binding properties of the actin-related protein Arp8 and its role in DNA repair. PLoS ONE 9:e108354. doi: 10.1371/journal.pone.0108354

Park, E. J., Hur, S. K., and Kwon, J. (2010). Human INO80 chromatin-remodelling complex contributes to DNA double-strand break repair via the expression of Rad54B and XRCC3 genes. Biochem. J. 431, 179-187. doi: 10.1042/BJ20100988

Ryter, S. W., Alam, J., and Choi, A. M. (2006). Heme oxygenase-1/carbon monoxide: from basic science to therapeutic applications. Physiol. Rev. 86, 583-650. doi: 10.1152/physrev.00011.2005 
Shen, X., Ranallo, R., Choi, E., and Wu, C. (2003). Involvement of actin-related proteins in ATP-dependent chromatin remodeling. Mol. Cell 12, 147-155. doi: 10.1016/S1097-2765(03)00264-8

Shimada, K., Oma, Y., Schleker, T., Kugou, K., Ohta, K., Harata, M., et al. (2008). Ino80 chromatin remodeling complex promotes recovery of stalled replication forks. Curr. Biol. 18, 566-575. doi: 10.1016/j.cub.2008.03.049

Tosi, A., Haas, C., Herzog, F., Gilmozzi, A., Berninghausen, O., Ungewickell, C., et al. (2013). Structure and subunit topology of the INO80 chromatin remodeler and its nucleosome complex. Cell 154, 1207-1219. doi: 10.1016/j.cell.2013.08.016

Vile, G. F., Basu-Modak, S., Waltner, C., and Tyrrell, R. M. (1994). Heme oxygenase 1 mediates an adaptive response to oxidative stress in human skin fibroblasts. Proc. Natl. Acad. Sci. U.S.A. 91, 2607-2610. doi: 10.1073/pnas.91.7.2607

Wu, S., Shi, Y., Mulligan, P., Gay, F., Landry, J., Liu, H., et al. (2007). A YY1-INO80 complex regulates genomic stability through homologous recombinationbased repair. Nat. Struct. Mol. Biol. 14, 1165-1172. doi: 10.1038/nsmb1332

Yao, W., Beckwith, S. L., Zheng, T., Young, T., Dinh, V. T., Ranjan, A., et al. (2015). Assembly of the Arp5 (Actin-related Protein) subunit involved in distinct INO80 chromatin remodeling activities. J. Biol. Chem. 290, 25700-25709. doi: 10.1074/jbc.M115.674887

Yao, W., King, D. A., Beckwith, S. L., Gowans, G. J., Yen, K., Zhou, C., et al. (2016). The INO80 complex requires the Arp5-Ies6 subcomplex for chromatin remodeling and metabolic regulation. Mol. Cell. Biol. 36, 979-991. doi: 10.1128/MCB.00801-15

Yao, T., Song, L., Jin, J., Cai, Y., Takahashi, H., Swanson, S. K., et al. (2008). Distinct modes of regulation of the Uch37 deubiquitinating enzyme in the proteasome and in the Ino80 chromatin-remodeling complex. Mol. Cell 31, 909-917. doi: 10.1016/j.molcel.2008.08.027

Zhang, J., Ohta, T., Maruyama, A., Hosoya, T., Nishikawa, K., Maher, J. M., et al. (2006). BRG1 interacts with Nrf2 to selectively mediate HO-1 induction in response to oxidative stress. Mol. Cell. Biol. 26, 7942-7952. doi: 10.1128/MCB.00700-06

Conflict of Interest Statement: The authors declare that the research was conducted in the absence of any commercial or financial relationships that could be construed as a potential conflict of interest.

Copyright (c) 2017 Takahashi, Murakami, Akiyama, Katoh, Oma, Nishijima, Shibahara, Igarashi and Harata. This is an open-access article distributed under the terms of the Creative Commons Attribution License (CC BY). The use, distribution or reproduction in other forums is permitted, provided the original author(s) or licensor are credited and that the original publication in this journal is cited, in accordance with accepted academic practice. No use, distribution or reproduction is permitted which does not comply with these terms. 